\title{
Protée
}

\section{Présentation : Sémiotique et bouddhisme. Quelques repères}

\section{Louis Hébert}

Volume 39, numéro 2, automne 2011

URI : https://id.erudit.org/iderudit/1007163ar

DOI : https://doi.org/10.7202/1007163ar

Aller au sommaire du numéro

Éditeur(s)

Département des arts et lettres - Université du Québec à Chicoutimi

ISSN

0300-3523 (imprimé)

1708-2307 (numérique)

Découvrir la revue

Citer ce document

Hébert, L. (2011). Présentation : Sémiotique et bouddhisme. Quelques repères. Protée, 39(2), 5-8. https://doi.org/10.7202/1007163ar d'utilisation que vous pouvez consulter en ligne.

https://apropos.erudit.org/fr/usagers/politique-dutilisation/ 


\title{
Sémiotique et bouddhisme Quelques repères
}

\author{
LOUIS HÉBERT
}

\begin{abstract}
A u PREMIER ABORD, on pourrait voir dans le thème proposé une exploitation mécanique de la capacité du «et» de conjoindre toutes choses. Mais le bouddhisme et la sémiotique se relient de plusieurs manières fortes, parfois inattendues. Et si certains de ces liens pertinents ont commencé d'être explorés, la plupart restent encore à dégager.
\end{abstract}

Toute religion se pose la question du sens, immanent et transcendant, et par là pose des questions sémiotiques, définit des structures du signe, des typologies des sens, des processus et critères interprétatifs, etc. Il est donc une sémiotique bouddhiste comme il est, par exemple, une sémiotique chrétienne. En fait, puisque le bouddhisme est particulièrement protéiforme, il est de nombreuses sémiotiques bouddhistes. Par exemple, pour prendre simplement le problème de la référence:

\begin{abstract}
Dans le bouddhisme ancien, la vision karmique du monde ou saṃsāra résulte de la projection d'une interprétation égotique sur un arrière-monde neutre et objectif. L'école mahayaniste yogācāra et le vajrayāna vont plus loin : il n'existe pas d'arrière-monde et tous les phénomènes que nous ressentons sont de simples perceptions sans plus (sanskrit vijñaptimātra), surgies de l'esprit qui les prend pour référents. (Cornu, 2009: 26)
\end{abstract}

Les religions sont également des objets sémiotiques, parmi d'autres. Parmi d'autres mais, également, plus que d'autres, pour ainsi dire: elles sont, pour le meilleur et pour le pire, au fondement des cultures, même de celles qui, comme l'occidentale, réévaluent et parfois rejettent leur héritage religieux. L'étude des religions peut bien sûr porter sur les objets qu'elles produisent - textes, images, sculptures, monuments, livres, rituels, concepts, etc. - ou sur les performances qui les concrétisent. L'étude des textes religieux est assurément la plus répandue et la plus avancée.

L'étude sémiotique des textes chrétiens est courante et déjà ancienne. II n'est que de penser aux travaux du Centre pour l'analyse du discours religieux (Cadir, Université de Lyon) qui, malgré son nom, s'intéresse exclusivement aux lectures sémiotiques de la Bible. La revue qu'il anime, Sémiotique et Bible, est publiée depuis 1975. Au Québec, pensons au groupe Aster (Analyse sémiotique des textes religieux).

L'étude sémiotique des textes bouddhistes et, même plus largement, du bouddhisme sous quelque aspect que ce soit, reste, quant à elle, embryonnaire. Parmi les chercheurs pionniers, on trouve Roland Barthes (1970), François Rastier (2006) et, surtout, Fabio Rambelli. Ce dernier, actif dans le monde anglophone et italophone, s'avère, à notre connaissance, le seul sémioticien qui se consacre essentiellement à l'étude du bouddhisme. C'est dire que le dossier que nous proposons permettra de baliser quelque peu une terre quasi vierge, particulièrement pour ce qui est du monde francophone.

Plus fondamentaux et plus étonnants sont les liens entre le bouddhisme et la sémiotique de Saussure et, conséquemment, les sémiotiques saussuriennes, de Hjelmlsev à Rastier : 
Ne serait-ce que par ses études de sanscrit, commencées dès sa treizième année, puis sa formation d'indo-européaniste à Leipzig, Saussure avait naturellement été en contact approfondi avec la pensée indienne. [...] Nous soulignerons des affinités remarquables entre la critique saussurienne de I'ontologie aristotélicienne qui préside aux théories de la signification et la critique bouddhiste de I'ontologie des logiciens védiques. Nous ne chercherons pas cependant à déterminer si les rencontres entre Saussure et les penseurs bouddhistes se justifient par des "influences », ou reflètent simplement une logique des positions théoriques. (Rastier, 2006; texte en ligne)

Barthes, quant à lui, a donné une interprétation proprement sémiotique de concepts bouddhistes et «a contribué à la problématisation sémiotique de concepts, tels la vacuité et l'éveil, généralement envisagés d'un point de vue religieux» (Rambelli, texte en ligne; notre traduction) ${ }^{1}$.

D'autres auteurs non seulement se laissent influencer par le bouddhisme, mais produisent des syncrétismes en mélangeant concepts bouddhistes et sémiotiques. C'est ainsi que

Floyd Merrel (1991), utilisant le concept de la semiosis illimitée de Peirce comme point de départ, essaie de décrire une théorie sémiotique appropriée à la «nouvelle» cosmologie. Selon Merrell, qui décrit le cosmos comme un flux sémiosique incessant, il n'y a aucune façon de parler de la réalité objective parce que tout ce qui existe dans notre monde «ne peut être plus que sémiotiquement réel». Pour esquisser sa cosmologie sémiotique, Merrell recourt aussi aux métaphores bouddhistes et à des concepts comme la vacuité et le filet d'Indra. (Ibid.) ${ }^{2}$

Il existe également quelques liens entre la sémiotique et le bouddhisme qui proviennent d'une similitude plus aléatoire. On pourrait mentionner l'étonnante ressemblance - mais on sait que le simple hasard est l'un des plus grands pourvoyeurs de ressemblances - entre, respectivement, d'une part, priméité et secondéité de Peirce et, d'autre part, dans le bouddhisme, pensée non dualiste (le type de pensée auquel aspire le pratiquant) et pensée dualiste (le type de pensée ordinaire, qui mène à la confusion et donc à la création du samsāāra, c'est-à-dire à l'insatisfaction). Quant à nous, nous avons montré (Hébert, à paraître) les ressemblances et différences entre le carré sémiotique, le tétralemme des Grecs anciens et le catuşkoşi bouddhiste systématisé par Nāgārjuna. Ces derniers liens ne sont pas qu'aléatoires, puisque le carré sémiotique est partiellement dérivé du tétralemme et qu'Aristote connaît «parfaitement le tétralemme sinon le mot » de «catuşkoţi» (Bugault, $2002: 2821$ ).

Pour une approche plus complète des relations entre sémiotique et bouddhisme, on consultera en ces pages I'article de Fabio Rambelli, spécialiste de la question. Nous nous contenterons ici, puisque le bouddhisme est à la fois tellement à la mode, si l'on peut dire, et si mal connu, de définir sommairement les traits principaux d'un objet bouddhiste et de signaler quelques liens entre ces traits et la sémiotique ou des sémiotiques spécifiques.

Il est extrêmement difficile de présenter une synthèse du bouddhisme, même en quelques centaines de pages. Il y a à cela plusieurs raisons, dont les principales sont sans doute: I'amplitude temporelle; l'étendue géographique; la variété linguistique et culturelle; le nombre et la diversité des textes canoniques (par exemple, le Kangyour, le canon tibétain, compte 100 volumes) et des pratiques qui y sont associées. Qu'y a-t-il de commun entre les trois

1. "Barthes acontributed to the semiotic problematization of concepts such as emptiness and enlightenment, usually considered only from a religious point of view."

2. "Floyd Merrel (1991), using Peirce's theme of unlimited semiosis as a starting point, attempts to outline a theory of semiotics suitable to the 'new' cosmology. According to Merrell, who describes the cosmos as an incessant semiosic flow, there is no way to talk about objective reality because e'verything that exists in our world 'can be no more than semiotically real.' To sketch his semiotic cosmology, Merrell resorts also to Buddhist metaphors and concepts such as emptiness and Indra's net." 
grandes formes du bouddhisme, si différentes, que sont I'hĩnayāna (le theravāda est la seule école actuellement existante issue du bouddhisme de ce courant), le mahāyāna (dont la forme la plus connue est sans doute le zen) et le vajrayāna (ou tantrisme bouddhiste, à distinguer du tantrisme hindou)? Cornu (2004) identifie trois traits communs principaux: la prépondérance accordée à la grande compassion et à la sagesse (qualités que nous définirons plus loin); un acte de foi et de pratique - la prise de refuge dans les trois joyaux, soit le Bouddha, le dharma (la doctrine) et la sangha (la communauté des pratiquants) -; les «quatre sceaux des préceptes». Ces «quatre sceaux des préceptes» sont quatre grandes thèses que, en principe, toute production bouddhiste (texte, concept, représentation, etc.) véhicule, ou à tout le moins présuppose.

(1) Tous les phénomènes conditionnés (et donc composés) sont impermanents: «Ils naissent quand les conditions sont réunies et se dissolvent lorsque celles-ci cessent d'être présentes» (ibid. : 15). L'impermanence grossière, celle des journées, des saisons, par exemple, est patente. Mais tout ce qui est change à chaque instant. C'est dire qu'une sémiotique dynamique (la sémiotique tensive, par exemple), qui met l'accent sur les processus plutôt que sur les états, toujours transitoires, est nécessaire pour appréhender correctement le monde (dans la mesure où l'on considère qu'une théorie doit épouser plus ou moins la forme de l'objet qu'elle veut décrire).

(2) Tout ce qui est corrompu est souffrance: «ce qui est conditionné est précisément corrompu. Mais la corruption réside surtout dans nos perceptions illusionnées [fruit de l'ignorance] qui attribuent une permanence à ce qui n'en a pas et une réalité à ce qui n'est qu'illusion. L'illusion est un décalage par rapport à la réalité » (ibid.). Ce décalage ne peut mener, encore une fois, qu'à la souffrance. "Souffrance», dans le bouddhisme, doit être entendue au sens large de «dysphorie». Cette souffrance au sens large englobe donc la souffrance au sens étroit, mais également toute forme d'insatisfaction, de malaise, etc. L'ignorance entraîne la production d'émotions ou de passions négatives, les deux principales étant l'attachement (entendu au sens d'attachement égotique malsain) et l'aversion (dont les expressions les plus fortes sont la haine et la colère). La sémiotique thymique ou axiologique et la sémiotique des passions seront particulièrement pertinentes pour décrire les objets et processus euphoriques et dysphoriques abordés dans le bouddhisme.

(3) Tous les phénomènes sont dépourvus de soi : «s'ils sont conditionnés, les phénomènes sont donc dépendants de causes et de circonstances liées à d'autres phénomènes tout aussi conditionnés. [...] Pourtant, nous ne cessons pas d'attribuer aux êtres et aux choses une identité permanente et autonome» (ibid. : 15-16). Cette méprise, comme toute méprise, ne peut que conduire à la souffrance. La valeur saussurienne (dans la langue, il n'y a que des différences) peut être vue comme une transposition dans la langue de l'interdépendance même régissant le samsāra, notre monde conditionné.

(4) Le nirvāṇa est paix:

Si le Bouddha n'avait enseigné que les trois marques de l'existence [les trois premiers sceaux], le bouddhisme pourrait effectivement être taxé de religion pessimiste comme l'ont cru bon nombre de ses détracteurs [...]. Loin d'être un anéantissement comme l'ont cru nombre de penseurs occidentaux, le nirvāṇa désigne un état de liberté complète où l'on est affranchi définitivement de tout conditionnement et de toute souffrance. Comment, cependant, décrire l'inconditionné si ce n'est en des termes négatifs par rapport aux phénomènes conditionnés, les seuls que nous connaissons dans ce monde? (Ibid.)

La cognition de l'être éveillé est au-delà de la pensée conceptuelle, toujours duelle, et l'Éveil, en tant qu'objet ou en tant qu'expérience, ne peut être proprement décrit par la pensée conceptuelle. Notamment, il ne se laisse pas catégoriser sous l'une ou l'autre des quatre positions et propositions du tétralemme (ou des dix positions et propositions du carré sémiotique - voir Hébert, à paraître). On ne peut donc y appliquer aucune des proposi- 
tions suivantes, dont l'ensemble est réputé exhaustif: cela est; cela n'est pas; cela est et n'est pas; ni cela est ni cela n'est pas. Cette indécidabilité absolue, conséquence de l'indétermination absolue de l'objet, n'est pas sans lien, comme nous l'avons dit, avec la priméité peircienne.

Notre regard portera dans trois directions principales, qui seront autant de volets de ce numéro: bouddhisme et théories sémiotiques; bouddhisme et interprétation; représentations bouddhistes.

Dans le premier volet, Fabio Rambelli développe une synthèse des relations avérées ou à développer entre bouddhisme et sémiotique. Il approfondit ces relations chez Barthes et dans une stratégie de remotivation des signes dans le shingon. Sungdo Kim traite de la rencontre entre le bouddhisme et, d'une part, Saussure et, d'autre part, Lévi-Strauss; en particulier, les liens entre ce dernier et le bouddhisme n'ont jamais, semble-t-il, été étudiés avant. Les confluences entre bouddhisme et neurosémiotique, et plus généralement science, sont l'objet du texte de Daniel S. Larangé; le postulat de base, très simple mais capital dans le bouddhisme, en est que la manière de penser le réel détermine la façon de le vivre.

Dans le cadre du deuxième volet sur le bouddhisme et l'interprétation, Anna Ghiglione étudie la fonction sémiotique des images langagières dans la version chinoise du Sūtra du Lotus. Simon Kim, quant à lui, touche au signe et à la «destruction» du sens dans le kōan zen. Les deux articles montrent les stratégies visant, paradoxalement, à utiliser le langage pour dépasser la pensée conceptuelle dyadique qu'il charrie et atteindre à la cognition adéquate.

Pour terminer, au sein du volet sur les représentations bouddhistes, Lionel Obadia discute de l'« être bouddhiste» en contexte occidental, où sont brouillées les marques traditionnelles d'adhésion relevant de signes comportementaux, discursifs ou vestimentaires. Louis Hébert explore les opérations de transformation dans un corpus iconographique du bouddhisme tibétain; il appert que les opérations d'adjonction et d'augmentation sont privilégiées dans les représentations, au détriment de la suppression et de la diminution. Benoît Mauchamp, enfin, s'attarde au concept sémiotique de l'empreinte, en particulier tel qu'il se manifeste dans les films Little Buddha et

Unmistaken Child; I'empreinte est l'inscription des formes signifiantes, ici la conscience d'un être réalisé décédé, dans un substrat matériel, ici un nouveau corps.

\section{OUVRAGES CITÉS}

BARTHES, R. [(1970) 2007] : L'Empire des signes, Paris, Seuil.

BuGault, G. [(1990) 2002] : «Catuşkoşti», dans S. Auroux (dir.), Les Notions philosophiques : dictionnaire, Paris, PUF, 28202821.

CORNU, P. [2004] : «Quelle unité sous la diversité des expressions?» dans P. Cornu et M. Gotin, La Terre du Bouddha, Paris, Seuil, 14-17;

[2009] : «Introduction », dans Padmasambhava, Le livre des morts tibétain, Paris, Buchet-Chastel, 19-55.

HÉBERT, L. [2007] : "L'analyse thymique», Dispositifs pour l'analyse des textes et des images. Introduction à la sémiotique appliquée, Limoges, Pulim, 151-172;

[à paraître] : «Sémiotique et bouddhisme. Carré sémiotique et tétralemme (catuşkotii) », dans L. Hébert et L. Guillemette (dir.), Performances et objets culturels, Québec, Presses de I'Université Laval.

MerReLL, F. [1991] : Signs Becoming Signs. Our Perfusive, Pervasive Universe, Bloomington, Indiana University Press.

RAmBeLLI, F. [s.d.] : Buddhism. En ligne: http://psychology.jrank.org/pages/1949/Buddhism.html (page consultée le 12 mai 2011).

RASTIER, F. [2006] : «Saussure, la pensée indienne et la critique de l'ontologie», Texto!. En ligne: http://www.revue-texto.net/ Saussure/Sur_Saussure/Rastier_Inde.html (page consultée le 12 mai 2011). Texte d'abord paru en 2002 dans la Revue de sémantique et de pragmatique, $\mathrm{n}^{\circ} 11,123-146$. 